\title{
Compruebe sus conocimientos sobre las técnicas de planíficación (II)
}

Margarita Peya Gascóns

Profesora titular. Administración Servicios de Enfermería. Escuela de Enfermería de la Universidad de Barcelona. Barcelona. España.

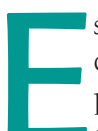
ste artículo es la segunda parte del publicado en el anterior número de Nursing. Continuamos poniendo de relieve la importancia que tienen las técnicas de planificación para realizar trabajos o proyectos participativos que lleven a la prevención, el análisis y la resolución de problemas, desde la perspectiva de una buena planificación. Como comentábamos en la primera parte, estas técnicas también se utilizan en las metodologías de mejora de la calidad asistencial.

1. Los árboles de decisiones son:

a. Diagramas que ayudan a tomar la decisión más adecuada ante un abanico de posibles decisiones.

b. Una forma gráfica y analítica de representar una serie de acontecimientos que pueden desarrollarse cuando se han de tomar decisiones.

c. Formas gráficas que se componen de nódulos de decisiones y probabilidades.

d. Todas son ciertas.

2. El método de Hanlon:

a. Es un método que ayuda en el establecimiento de prioridades de salud.

b. Es un método de priorización basado exclusivamente en la gravedad de los problemas sanitarios.

c. Es una técnica de evaluación fundamentada únicamente en la magnitud de los problemas que se presentan.

d. Es un método de valoración que plantea diferentes alternativas previamente seleccionadas.

3. Los cuatro componentes en los que se basa el método de Hanlon para la priorización son los siguientes:

a. Gravedad del problema, facilidad en su medición, coste de la mejora, influencia externa.

b. Problemática sanitaria, factores de riesgo, gravedad del problema, eficacia de la solución. c. Magnitud del problema, severidad, eficacia de la solución, factibilidad de la intervención o programa.

d. Severidad del problema, factores de riesgo, coste de la mejora, factibilidad de la intervención.

4. Para valorar el componente "factibilidad de la intervención o programa" del método de Hanlon, se plantea:

a. Analizar la aceptabilidad, la legalidad, el coste económico y la extensión de los problemas que se plantean para su valoración.

b. Analizar la pertinencia, la factibilidad económica, la aceptabilidad, la disponibilidad de recursos y la legalidad de los problemas que se plantean para su priorización.

c. Analizar el valor relativo y total de cada alternativa seleccionada por los miembros del grupo de estudio.

d. a y c son ciertas.

5. Las siglas DAFO CAME de la técnica de planificación estratégica significan:

\begin{tabular}{lll}
\hline a. & Documentos & Calidad \\
& Algoritmos & Alinear \\
& Frecuencias & Mitigar \\
& Observaciones & Evaluar \\
\hline b. & Documentación & Control \\
& Ansiedades & Afrontar \\
& Formación & Mantener \\
& Objetivos & Examinar \\
\hline
\end{tabular}

\begin{tabular}{lll}
\hline c. & Docencia & Calidad \\
& Abstracciones & Abreviar \\
& Formación & Mantener \\
& Objetivos & Evaluar \\
\hline d. & Debilidades & Combatir \\
& Amenazas & Afrontar \\
& Fortalezas & Mantener \\
& Oportunidades & Explorar \\
\hline
\end{tabular}

6. La técnica DAFO CAME se utiliza para realizar:

a. Un análisis del coste efectividad de los procedimientos asistenciales de una institución.

b. Un análisis de los entornos interno y externo de una institución.

c. Una evaluación exhaustiva de la eficacia y de la eficiencia de una institución.

d. Una evaluación del contexto interno de una institución.

7. En la técnica DAFO CAME, combatir las debilidades y mantener las fortalezas corresponden a la etapa del:

a. Análisis del entorno externo de una institución.

b. Análisis del entorno interno de una institución.

c. Análisis de los entornos tanto interno como externo.

d. a y b son ciertas.

8. En la técnica DAFO CAME, afrontar las amenazas y explorar las oportunidades corresponde a la etapa del:

a. Análisis del entorno externo de una institución. 


\section{Did \\ edición española}

b. Análisis del entorno interno de una institución.

c. Análisis de los entornos tanto interno como externo.

d. a y b son ciertas.

\section{La técnica DAFO CAME}

proporciona una buena base para:

a. Analizar la situación actual del centro con sus métodos y procedimientos.

b. Analizar el desarrollo de nuevos servicios.

c. Analizar la oportunidad para realizar una adquisición/inversión.

d. Todas son ciertas.

10. Cuando se quiere realizar un análisis DAFO, es conveniente

utilizar una plantilla que consiste en:

a. Una matriz con cuatro secciones en la que se describen:

\begin{tabular}{l|l}
\hline Debilidades & Fortalezas \\
\hline Amenazas & Oportunidades \\
\hline
\end{tabular}

b. Una matriz con seis secciones en la que se describen:

\begin{tabular}{l|l|l}
\hline Debilidades & Fortalezas & Estructura \\
\hline Amenazas & Oportunidades & Recursos \\
\hline
\end{tabular}

c. Una matriz con ocho secciones en la que se describen:

\begin{tabular}{c|c|c|c}
\hline Debilidades & Fortalezas & Oportunidades & Amenazas \\
\hline Deblinas
\end{tabular}

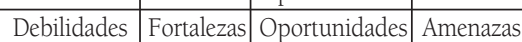
\begin{tabular}{l|l|l|l} 
externas & externas internas & internas \\
\hline
\end{tabular}

d. a y c son ciertas.

11. El diagrama de Gantt es:

a. Una herramienta que explica las relaciones existentes entre las diferentes actividades a realizar de un proyecto y durante un período de tiempo determinado.

b. Un instrumento que representa la magnitud real de un proyecto o el tamaño relativo de los elementos del proyecto a realizar.

c. Una herramienta que sirve para mostrar el tiempo de dedicación previsto para realizar las diferentes actividades de las que consta un proyecto, a lo largo de un período de tiempo determinado.

d. a y b son ciertas.
12. Indica cuál de los siguientes gráficos es un diagrama de Gantt:

a.

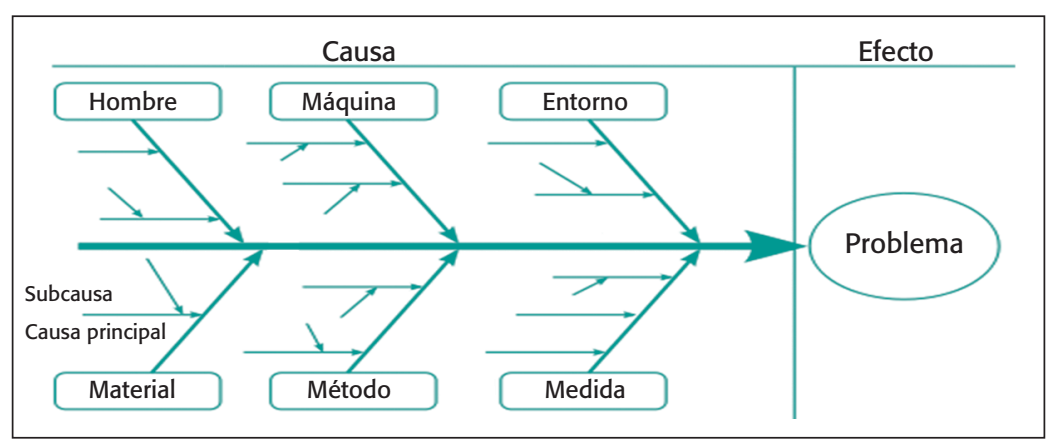

b.

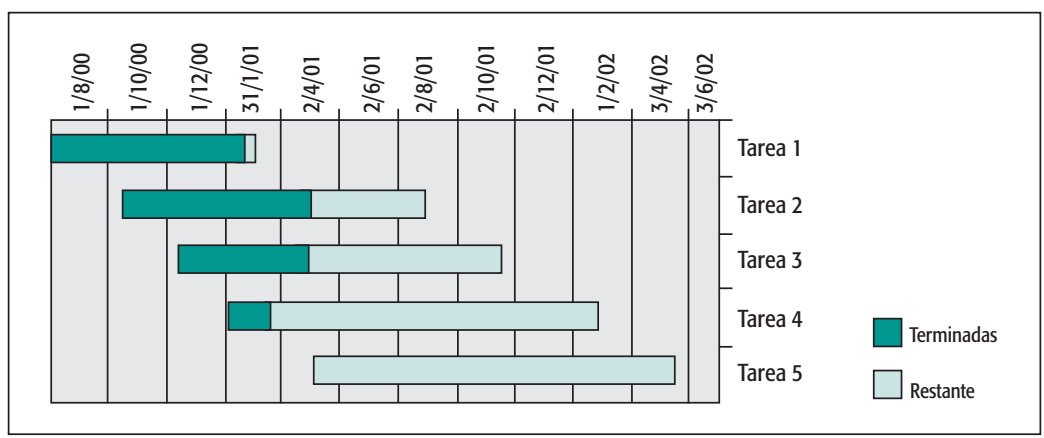

c.

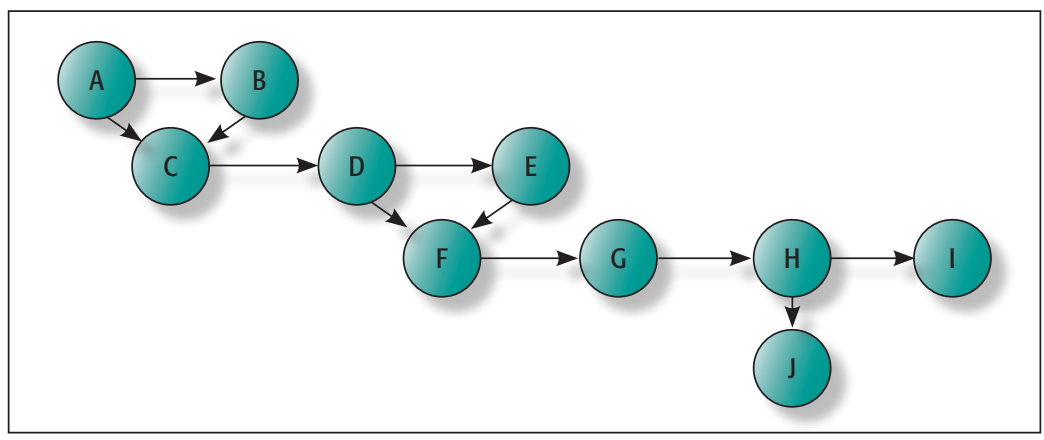

d.

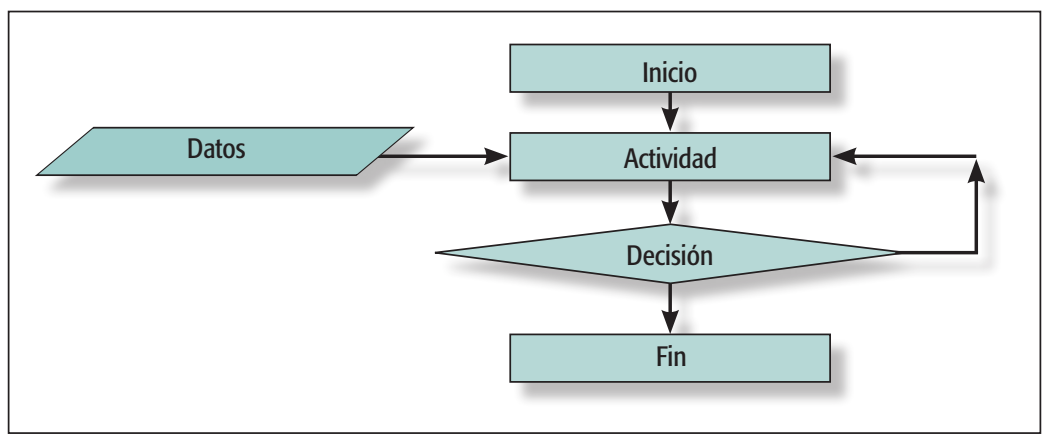




\section{Respuestas}

1. d. Los árboles de decisiones nos ayudan, como indica su nombre, a tomar la mejor decisión, y ante un conjunto de alternativas nos sirven de guía.

2. a. El método de Hanlon sirve para el establecimiento de prioridades y se basa en cuatro criterios para establecer dicha priorización. Las respuestas b, c y d no responden a este método, tal como están formuladas.

3. c. Los cuatro componentes que plantea Hanlon se encuentran en esta respuesta. Las otras respuestas no son válidas, ya que responden a factores aislados que plantean otros métodos de priorización.

4. b. Los componentes que plantea esta respuesta son los correctos en referencia a la valoración del componente "factibilidad de la intervención", de acuerdo al método de Hanlon. Las demás respuestas son incompletas y responden a otras técnicas de priorización de problemas.

5. d. Ésas son el verdadero significado de las siglas CAME DAFO, como técnica de planificación estratégica. Las demás respuestas son pura invención.
6. b. La técnica CAME DAFO analiza el contexto interno (comprende las áreas clave internas de la institución sobre las que se tiene control) y el entorno externo (áreas clave externas de la institución, sobre las que no se tiene control).

7. b. En el análisis interno se analizan los aspectos de la institución que se deben mantener (fortalezas) o minimizar (debilidades) para afrontar los retos que plantea el entorno externo. Son variables que controla la organización.

8. a. El objetivo fundamental del análisis externo es identificar y prever la evolución y los cambios que existen en dicho entorno; esto significa identificar las amenazas y explorar las oportunidades de desarrollo. Son variables no controlables.

9. d. Tiene múltiples aplicaciones, entre las que figuran las que hemos nombrado. 10. a. Para realizar un análisis $D A F O$ de una institución se debe utilizar una matriz de este tipo, con cuatro secciones. Las otras dos contienen conceptos erróneos.

11. c. El diagrama de Gantt muestra la dedicación en tiempo de las diferentes partes de un proyecto.
12. b. En el diagrama de Gantt siempre constan las actividades de un proyecto y el período de tiempo para realizarlas. La respuesta a responde a un diagrama causaefecto de Ishikawa. La respuesta c, a un diagrama de red Pert, y la respuesta d, a un diagrama de flujo.

\section{Bibliografía general}

Alward RR, Camuñas C. The nurse's guide to marketing. Washington DC: Delamr Publishers; 1991.

Huglo PE. Les cercles de qualité a l'hôpital. Paris: BergerLevarault; 2002.

Kunkle V. Marketing strategies for nurse managers. A guide for developing and implementing a nursing marketing plan. Maryland: Aspen Publishers; 1990.

Monteil B, Perigord M, Raveleau G. Les outils des cercles et de l'amélioration de la qualité. Paris: Les Editions d'Organisation Management 2000; 1999.

Peltier J, Nill A, Schibrowsky JA. Internal marketing, nurse loyalty and relationship marketing: an exploratory study of German nurses. Health Marq Q. 2003;20:63-82. Pineault R, Daveluy C. La planificación sanitaria. Conceptos, métodos y estrategias Barcelona: Masson; 1989 